\title{
Sculpting sandcastles grain by grain: Self-assembled sand towers
}

\author{
F. Pacheco-Vázquez, F. Moreau, N. Vandewalle, and S. Dorbolo \\ GRASP, Physics Department B5, Université de Liège, B4000-Liège, Belgium
}

(Received 30 May 2012; published 9 November 2012)

\begin{abstract}
We study the spontaneous formation of granular towers produced when dry sand is poured on a wet sand bed. When the liquid content of the bed exceeds a threshold value $W^{\star}$, the impacting grains have a nonzero probability to stick on the wet grains due to instantaneous liquid bridges created during the impact. The trapped grains become wet by the capillary ascension of water and the process continues, giving rise to stable narrow towers. The growth velocity is determined by the surface liquid content which decreases exponentially as the tower height augments. This self-assembly mechanism (only observed in the funicular and capillary regimes) could theoretically last while the capillary rise of water is possible; however, the structure collapses before reaching this limit. The collapse occurs when the weight of the tower surpasses the cohesive stress at its base. The cohesive stress increases as the liquid content of the bed is reduced. Consequently, the highest towers are found just above $W^{\star}$.
\end{abstract}

DOI: 10.1103/PhysRevE.86.051303

PACS number(s): 45.70.-n, 05.65.+b, 47.55.nb, 47.55.nk

\section{INTRODUCTION}

From sugar poured into a bowl to tons of grains discharged in industrial processes, a peculiar feature of granular materials emerges: the formation of a pile. When the grains are not cohesive, only friction and grain geometry determine the shape of the assembly which resembles a conical pyramid [1-3]. As known by any sandcastle architect, the addition of some liquid induces cohesion between the grains due to surface tension and capillary effects [4-12]. Recent studies demonstrate that the angle of repose of a wet pile initially increases with the liquid content, but afterward saturates. Similarly, the mechanical properties of the wet pile (tensile strength, yield stress, etc.) are remarkably insensitive to the amount of liquid over a wide range [4-6,8]. These facts reflect that the cohesive strength between grains increases from zero and becomes constant rapidly, at a very low liquid content $[6,10]$. This behavior is explained by a particular organization of liquid bridges among the grains into open structures (first trimers, then pentamers, and so on), up to forming large liquid clusters, such that the projected area of the particle over which the Laplace pressure acts, i.e., the cohesive force, reaches a constant value [10]. When the granular material is completely saturated with liquid, cohesion becomes negligible again as in the case of a dry pile.

Clearly, the level of liquid saturation in the granular material plays a key role. Depending on this saturation, four regimes of liquid content have been identified [11,13,14]: pendular (the grains are held together by liquid bridges and open structures appear), funicular (large liquid clusters and voids filled with air coexist), capillary (all voids are filled with liquid, and the grains are held together by capillary pressure), and slurry (grains fully immersed in liquid). Most of the experiments on the stability of wet piles have been carried out in the pendular regime $[4-6,8-10,15,16]$. It is known that the stability of the pile is unaffected by the presence of the fluid in the slurry regime [6]. On the other hand, the funicular and capillary regimes remain largely unexplored [11].

To introduce the phenomenon reported in this paper, let us analyze a simple experiment illustrated in Figs. 1(a)-1(c). When dry sand is poured on a dry plate, a characteristic conical pyramid is observed [Fig. 1(a)]. If the experiment is repeated over a pool of water, a conical pile is also formed [Fig. 1(b)]. But what happens when the pile reaches the water-air interface? We found that an intriguing phenomenon occurs: From that moment, the dry grains hit a wet sand surface and start to accumulate at the top of the heap, the angle of repose reaches $90^{\circ}$, and a vertical sand tower emerges [Fig. 1(c)]. In this paper, we study the growth dynamics and stability of these self-assembled structures. We find that the growth velocity of the tower decreases exponentially with its height until, abruptly, the structure collapses. The final height of the tower increases when the amount of liquid at its base is reduced. We deduce that the towers only arise in the funicular and capillary regimes when three conditions are satisfied: (i) The dry grains can be captured by capillary bridges, (ii) the liquid can be sucked up into the structure, and (iii) the weight of the tower has to be smaller than the cohesive stress at its base which is fixed by the local liquid content. The first two surmises concern the condition for growth while the third concerns the stability of the structure.

In a recent study reported by Chopin and Kudrolli [18] a granular suspension was deposited drop by drop on a dry substrate. The droplets, used as bricks, were superposed to give rise to a rich array of delicate vertical structures. In the present paper only dry sand is poured to build the towers. The growth dynamics is regulated by the liquid suction and trapping of grains, and the height of the structures characterizes the liquid content of the substrate. Therefore, this unforeseen mechanism allows us to study the imbibition process and mechanical properties of wet granular materials.

\section{EXPERIMENTAL SETUP AND MEASUREMENTS}

To build the towers we used sand grains (200-600 $\mu \mathrm{m}$ in size and density $\rho_{g}=2.66 \mathrm{~g} / \mathrm{cm}^{3}$ ) poured at a constant flow over a wet granular substrate with a given liquid content $W$ (liquid volume divided by the total pore space between the grains). Even when the sand towers can grow on an extended sand bed [Fig. 1(d)], the accumulation of grains makes it difficult to control $W$ during the experiment. For this reason we used a narrow sand bed poured contained in a vertical tube of $12 \mathrm{~mm}$ diameter connected to a water reservoir. A 

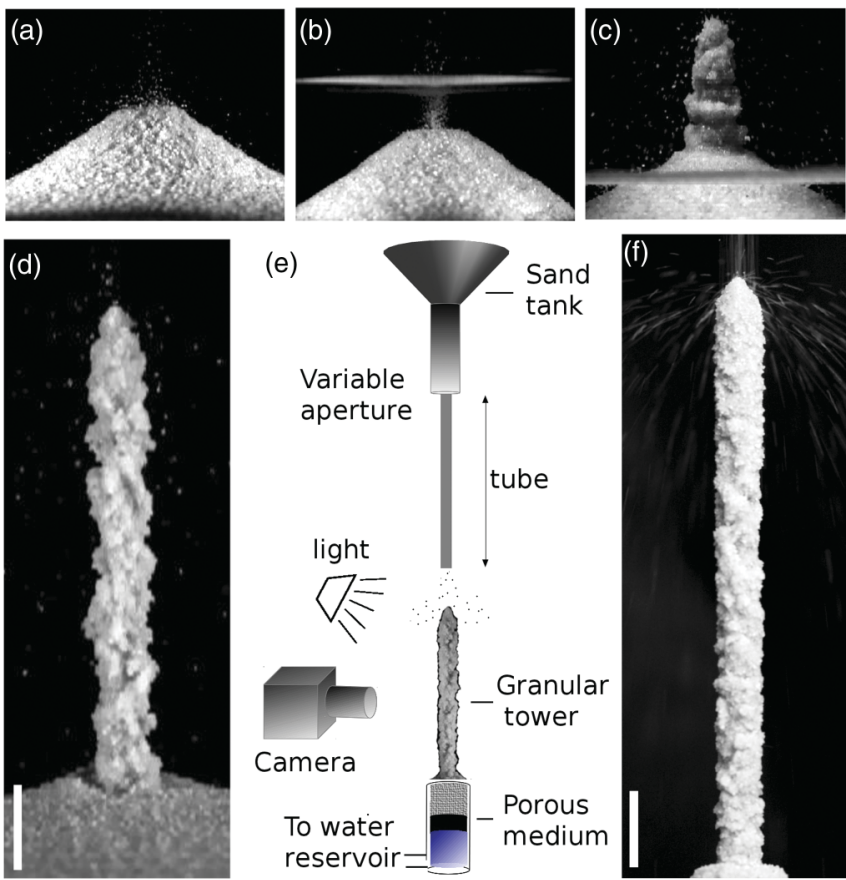

(e)
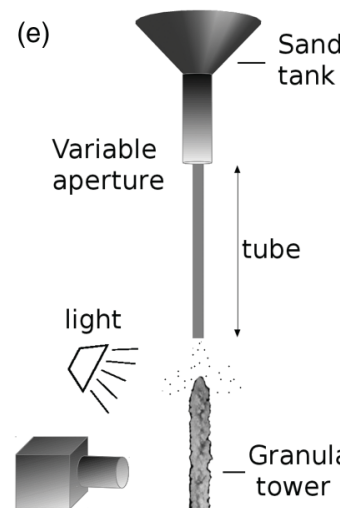

Camera

To water reservoir
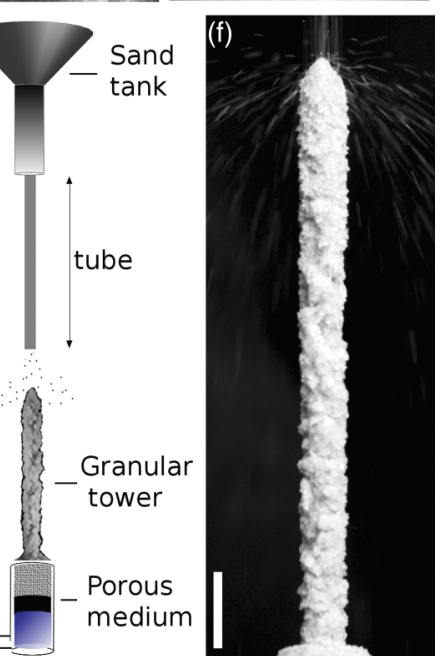

FIG. 1. (Color online) (a) Sand pile formed by pouring dry grains on a flat surface. (b) A similar pile is obtained when the grains are poured into water. (c) When the pile reaches the air-water interface a vertical structure starts to show up, see movie 1 [17]. (d) A stable sand tower growth onto a wet sand bed (movie 2 [17]). (e) Experimental setup used in our experiments. (f) Stable tower obtained using our experimental setup (movie 3 [17]). The scale bars correspond to $10 \mathrm{~mm}$.

porous medium was introduced into the tube at a distance $h_{s}$ from the top. The water level was adjusted so as to totally immersed the porous medium. The sand was poured until the tube was full. By changing the size of the sand layer $h_{s}$, it was possible to finely control $W$ at the surface of the sand in the range $0.78 \leqslant W \leqslant 1$, corresponding to $80 \leqslant h_{s} \leqslant 0 \mathrm{~mm}$, see details in the Supplemental Material [17]. Note that $W=1$ is equivalent to the water-air interface conditions described in Fig. 1(c). Using this method, the packing fraction of the sand substrate was $\phi_{s}=0.58 \pm 0.01$.

The sand was poured using a cylindrical silo with a variable aperture at the bottom that allowed us to obtain five different flux values $(Q=0.166,0.335,0.850,1.337$ and $3.6 \mathrm{~g} / \mathrm{s})$. A glass tube of $6 \mathrm{~mm}$ inner diameter and $400 \mathrm{~mm}$ long was joined to the hole to collimate the flow of grains. The system was vertically aligned at $20 \mathrm{~mm}$ above the center of the substrate, as is shown in Fig. 1(e). The grains fell along the tube, reaching a terminal velocity $v_{g}=2073 \pm 28 \mathrm{~mm} / \mathrm{s}$ before they struck the bed (measured with a high speed camera). A movable support allowed us to displace the discharge system upward while the tower was growing, without affecting the impact velocity of the grains. By using this setup, stable and well-designed sand towers were obtained, see Fig. 1(f).

In a typical experiment, the sand bed liquid content was fixed, the flow was started, and the process filmed at $30 \mathrm{fps}$ until the tower collapsed. Figure 2(a) shows characteristic snapshots of the formation process (here, for $W=0.84$ and $Q=0.166 \mathrm{~g} / \mathrm{s}$ ), where a vertical tower is growing until it (a)

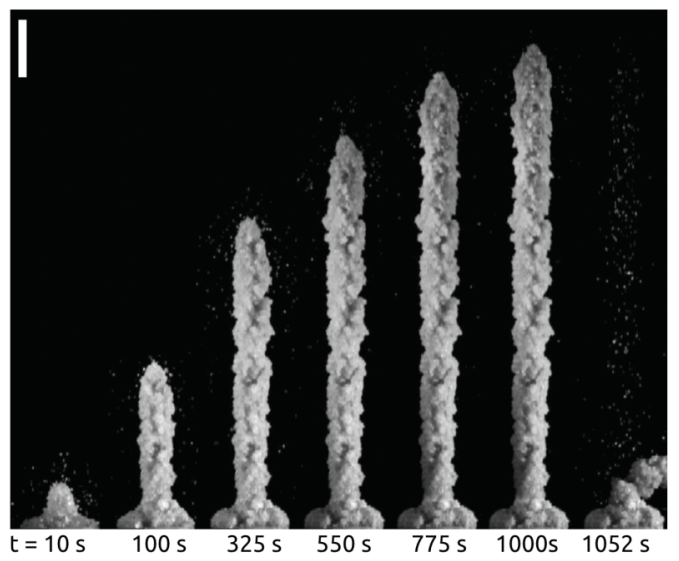

(b)

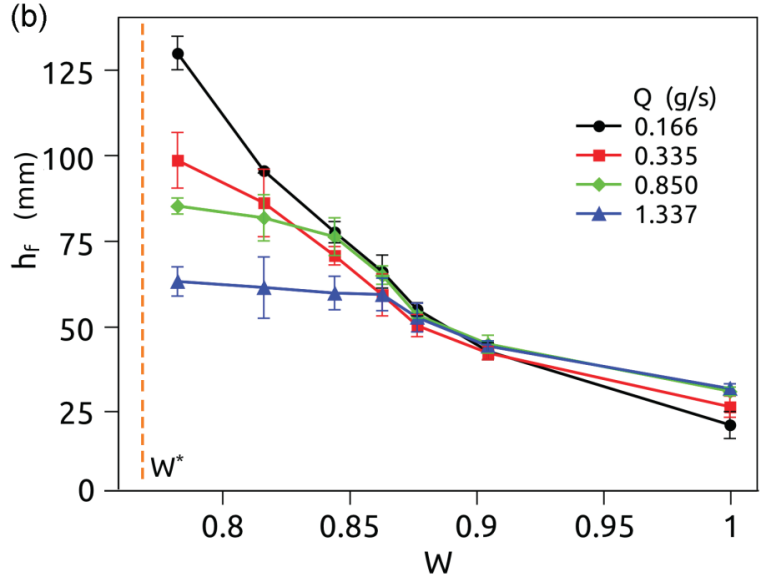

FIG. 2. (Color online) (a) Snapshots of a complete growing process. The average tower width is $6 \mathrm{~mm}$ and the scale bar is $10 \mathrm{~mm}$. (b) $h_{f}$ vs $W$ for the different flux values used in our experiments. Each point corresponds to five repetitions and the error bars to the standard deviation. For $W<W^{*}$ the grains cannot stick and sand towers do not emerge.

breaks at its base and falls (see movie 3 [17]). The videos were analyzed using IMAGEJ to obtain the evolution of the height $h(t)$, the final height before falling $h_{f}$, and the total growth time $t_{g}$. In Fig. 2(b) we plot $h_{f}$ vs $W$ for different values of $Q$. We can observe that the smaller the liquid content, the greater the height of the tower. On the other hand, $h_{f}$ decreases when the flux is increased. It is important to note that (i) if $W$ is smaller than a threshold value $W^{\star}$, the tower cannot growth, and (ii) the highest towers were obtained for $W$ slightly higher than $W^{\star}$. By performing several tests on wet substrates with the same packing fraction, we found that $W^{\star} \approx 0.72 \pm 0.04$. According to the literature $[11,13,14]$ this value indicates that the towers only arise in the funicular and capillary regimes.

To determine which are the crucial parameters that define the growth process, we plot in Figs. 3(a)-3(c) the height of the tower $h$ as a function of time $t$ and the growth velocity $v$ vs $h$, for experiments carried out at $Q$ constant [Figs. 3(a) and 3(b)] and $W$ constant [Fig. 3(c)]. In Fig. 3(a) the end of the trajectories represents the instant when the towers collapse (vertical lines). This occurs after almost $4 \mathrm{~h}$ for $W=0.78$ (see data in Supplemental Material [17]). The growth velocity data 

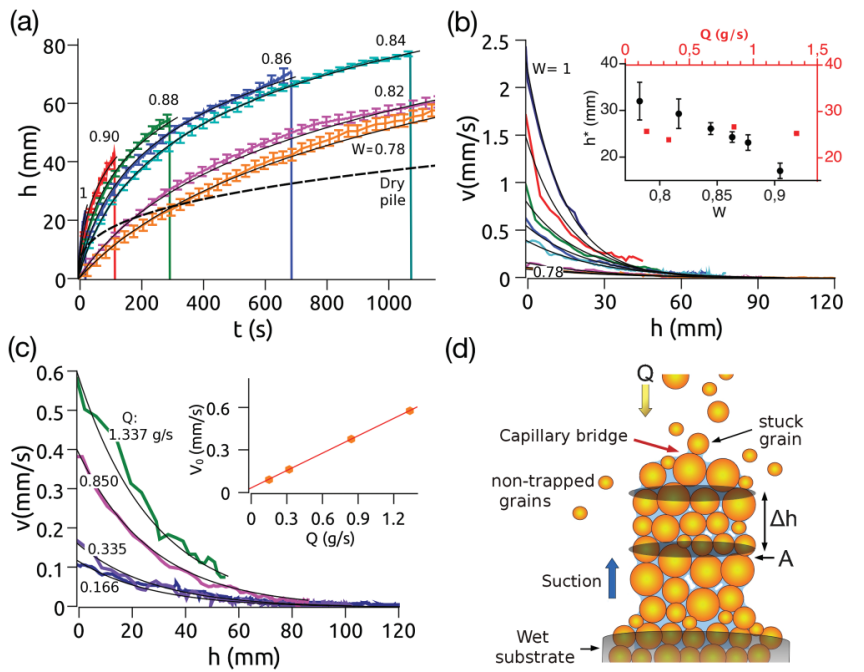

(d)

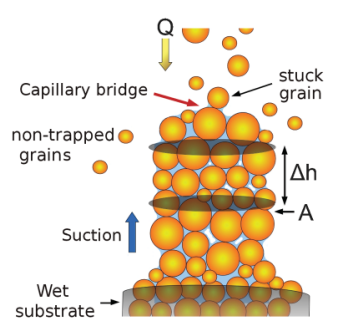

FIG. 3. (Color online) Growth dynamics: (a) $h$ vs $t$, and (b) $v$ vs $h$ obtained at constant flux $(Q=0.166 \mathrm{~g} / \mathrm{s})$ for different values of $W$ : dark blue line, 1 ; red, 0.90 ; green, 0.88 ; blue, 0.86 ; cyan, 0.84; magenta, 0.82; and orange, 0.78 . In (a) only the first $1200 \mathrm{~s}$ of growing are plotted. The evolution of a dry sand pile formed with the same flux and grain properties is compared (dashed line). Inset in (b): $h^{*}$ vs $W$ (black points) and $h^{*}$ vs $Q$ (red points). (c) $v$ vs $h$ at constant liquid content $(W=0.78)$ and different values of $Q$ : dark blue line, 0.166 ; violet, 0.335 ; red, 0.850 ; and green, $1.337 \mathrm{~g} / \mathrm{s}$. Inset: $v_{0}$ vs $Q$ for $W=0.78$. The orange line corresponds to $v_{0} / Q=(0.410 \pm 0.006) \mathrm{mm} / \mathrm{g}$. (d) Sticking and suction: only the grains impacting on wet sites can be trapped.

in Figs. 3(b) and 3(c) are well fitted by

$$
v(h)=v_{0} e^{-h / h^{*}},
$$

where $v_{0}$ is the initial growth velocity and $h^{*}$ a free parameter. From the fitting curves in Figs. 3(b) and 3(c) (black lines), we find that $h^{*}$ is independent on $Q$ and is only determined by $W$. By integrating $v(h)$, it is possible to obtain the growth dynamics law of $h$ as a function of $t: h(t)=h^{*}(W) \ln [1+$ $\left.v_{0} t / h^{*}(W)\right]$. This function is represented in Fig. 3(a) by continuous black lines.

\section{DISCUSSION}

From the observations, the tower grows when dry grains can be captured by wet grains. Then, it is required that the water is able to percolate by a suction mechanism up to the impacted surface. This suggests that $W^{\star}$ is the minimum liquid content to have liquid bridges interconnected through the granular material in order to maintain the imbibition process. Let us now study the connection between this suction process and the growth dynamics, what are the parameters that set the limit of imbibition (maximum reachable height), and finally, the collapse of the structure and why the highest towers are found at low liquid content, namely, close to $W^{\star}$.

\section{A. Growth dynamics}

To understand the growth dynamics of the towers, we propose the following model based on the probability for a dry grain to be trapped. Let us suppose that during a time
$\Delta t$ a volume of grains $V=\Delta m_{g} / \rho_{g}$ is discharged, some of them stick, occupying a volume $V^{\prime}=V \mathcal{P}(h) / \phi_{T}=\Delta h A$, where $\mathcal{P}(h)$ is the instantaneous probability of sticking, $\phi_{T}$ the packing fraction, and $A$ the horizontal cross section area of the tower, see the sketch in Fig. 3(d). The growth velocity can be expressed as a function of the sticking probability as $v(h)=\Delta h / \Delta t=Q \mathcal{P}(h) / \rho_{g} \phi_{T} A$, with $Q=\Delta m_{g} / \Delta t$. By comparing this expression with Eq. (1) we have that $\mathcal{P}(h)=$ $\mathcal{P}_{0} e^{-h / h^{*}}$ and $v_{0}=Q \mathcal{P}_{0} / \rho_{g} \phi_{T} A$. The linear dependence of $v_{0}$ with the flux is in good agreement with the experimental data shown in the inset in Fig. 3(c). Consequently, the speed of growth is given by

$$
v(h)=\frac{Q \mathcal{P}_{0}}{\rho_{g} \phi_{T} A} e^{-h / h^{*}(W)} .
$$

This expression indicates that $v(h)$ is proportional to the local sticking probability $\mathcal{P}(h)$. The change in $\mathcal{P}(h)$ during the growth process must be related to the liquid distribution along the tower. To quantify this distribution, a $90 \mathrm{~mm}$ sand tower (growth at $W=0.84$ and $Q=0.166 \mathrm{~g} / \mathrm{s}$ ) was segmented into small fragments of $10 \mathrm{~mm}$. The vertical position of each fragment measured from the substrate is given by $h^{\prime}$. The mass of the fragments was measured before and after drying to determine the amount of liquid. In Fig. 4(a) is shown $\omega=m_{l} / m_{s}$ vs $h^{\prime}$ ( $m_{l}$ and $m_{s}$ are the measured masses of liquid and of grains, respectively). An important gradient is observed from $\omega_{0}=0.225$ to $\omega=0.18$. After this value, $\omega$ slowly approaches a critical value $\omega^{\star}$. Actually, the local liquid content $\omega\left(h^{\prime}\right)$ is found to exponentially decrease towards $\omega^{\star}=0.175$. The data are well fitted by $\omega\left(h^{\prime}\right)=\omega^{\star}+\left[\omega(0)-\omega^{\star}\right] e^{-h^{\prime} / h^{\prime *}}$ (blue solid line), where $h^{* *}$ is a characteristic length. Leaving $h^{*}$ as a free parameter, one finds that $h^{\prime *}=23.5 \mathrm{~mm}$. This value is comparable to the characteristic length found in the growth dynamics law Eq. (2) for the same experimental conditions [inset of Fig. 3(b)]. This confirms the relation of $\mathcal{P}(h)$, and thus of $v(h)$, with the local liquid content of the material.

\section{B. Maximum reachable height}

According to the previous analysis, the limitation of the tower growth must be related to the maximum height $h_{\mathrm{imb}}$ that the water can reach by imbibition through the granular pores. With the assumption that the granular medium is composed by a complex entanglement of cylindrical capillary pipes of radius $r_{p}$ [19], the fluid flows upward until reaching $h_{\mathrm{imb}}=2 \gamma \cos \theta / \rho_{l} g r_{p}$, where $\gamma=0.072 \mathrm{~N} / \mathrm{m}$ is the surface tension and $\theta$ is the angle of contact of water on a glass grain. In the sand substrate inside the tube, $h_{\mathrm{imb}, s} \approx 90 \mathrm{~mm}$; see Supplemental Material [17]. On the other hand, we show in Fig. 2(b) that the highest tower can reach even $127 \mathrm{~mm}$. The difference should come from a decrease of the pore size in the tower, or in other words, to an increase of the volume fraction. To characterize this change in packing, the towers were weighed after drying for various sets of parameters. The mass of the dry tower $m_{T}$ is plotted as a function of its height $h_{f}$ in Fig. 4(b). By estimating the shape of the structure by a cylinder, we can express $m_{T}=\phi_{T} \rho_{g} \pi R_{T}^{2} h_{f}$, where $R_{T}$ is the radius of the tower. From Fig. 4(b) one finds that $\phi_{T}=0.66 \pm 0.03$, which is significantly larger than $\phi_{s}$. 

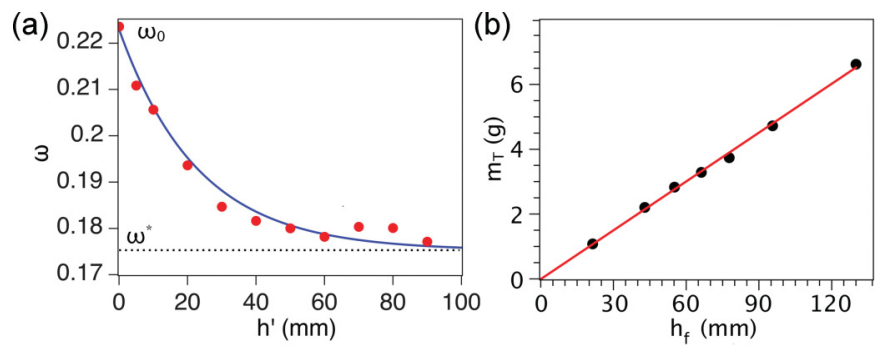

(c)
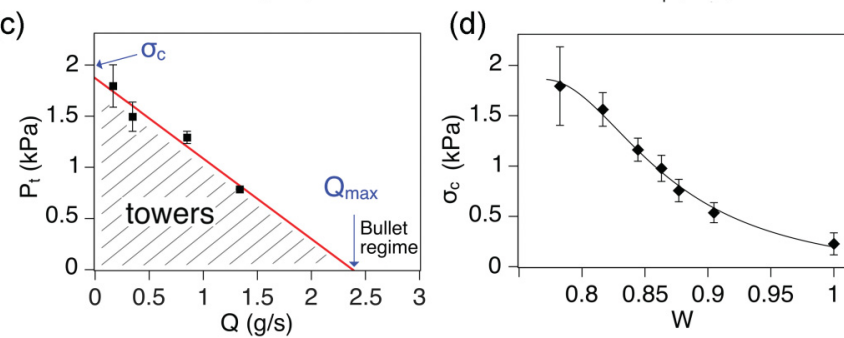

FIG. 4. (Color online) (a) $\omega=m_{l} / m_{s}$ vs $h^{\prime}$ : Experimental measurements (red points) and the best data fit (blue line), see text. (b) $m_{T}$ vs $h_{f}$ for the experiments shown in Fig. 2(b). $Q=0.166 \mathrm{~g} / \mathrm{s}$ is used because with this flux the erosion is negligible and the cylindrical shape can be perfectly assumed. The linear fit (red line) gives a slope $\phi_{T} \rho_{g} A_{T}=0.050 \pm 0.003$. (c) $P_{t}$ vs $Q$ for $W=0.78$. The critical values $\sigma_{c}=1.87 \mathrm{kPa}$ and $Q_{\max }=2.4 \mathrm{~g} / \mathrm{s}$ are indicated by the arrows. (d) $\sigma_{c}$ vs $W$ for $Q=0.166 \mathrm{~g} / \mathrm{s}$. The cohesive stress increases as the liquid content at the base of the tower decreases.

This larger value can be explained considering that the grain capture is the most efficient when the wet surface of contact is the largest. Consequently, the grains form a denser packing. Later in the text, we will see how this efficient trapping of grains ensures the maximum stability of the structure.

Now, let us estimate the maximum limit of imbibition for any cylinder of radius $R$ containing a granular material with volume fraction $\phi$ and pore size $r_{p}$. The cross section of the cylinder is $A=\pi R^{2}$. The area $A_{g}$ occupied by the grains is equal to $A \phi$. Then, the average number of grains $\mathcal{N}$ is obtained by $\mathcal{N}=A \phi / \pi \bar{r}_{g}^{2}$ where $\bar{r}_{g}$ is the average radius of a grain. When $\mathcal{N}$ is large, one can consider that we have $\mathcal{N}$ pores. The typical size of a pore is then given by $r_{p}=[(1-\phi) / \phi]^{1 / 2} \bar{r}_{g}$. Applied to our case, it is obtained that the ratio between the imbibition heights for the substrate and the tower is $h_{\mathrm{imb}, s} / h_{\mathrm{imb}, T}=\left[\left(1-\phi_{T}\right) \phi_{s} /\left(1-\phi_{s}\right) \phi_{T}\right]^{1 / 2} \approx$ $0.84 \pm 0.07$. Since $h_{\mathrm{imb}, s} \approx 90 \mathrm{~mm}$, the theoretical maximum height is $h_{\mathrm{imb}, T}=107 \pm 8 \mathrm{~mm}$. As a first approximation, this model predicts very well the size of the highest towers found experimentally. A more refined analysis must consider that the smaller grains have a higher probability of sticking than the larger ones. Therefore, the pores in the tower are smaller and the real imbibition height is greater.

\section{Mechanical limitation}

Our experimental results in Fig. 2(b) show that the towers collapse at a given $h_{f}$ depending on $Q$ and $W$, which is in most of the cases much smaller than the maximum reachable height. Then, an important question remains open: What is the mechanism that determines the final size of the tower? In other words, why do the towers fall? An important clue is that the towers always break at their base, where the liquid content is the highest (see movie 4 [17]). Thus, the mechanical properties of the wet sand located at the base of the tower are responsible for the stability of the edifice. The cohesive stress $\sigma_{c}$ which maintains the grains together must be higher than the vertical pressure. The total pressure is the sum of the pressure due to the tower, $P_{t}=m_{t} g / A$, and the pressure due to the grains that collide the top of the structure, $P_{g}=k Q v_{g} / A$, where $m_{t}$ is the mass of the wet tower and $k$ is a free parameter. Then, the condition of stability is

$$
P_{t}+\frac{k Q v}{A}<\sigma_{c} .
$$

The tower collapses when both sides balance. Figure 4(c) shows $P_{t}$ as a function of $Q$ for $W=0.78$ (this liquid content allows us to explore the widest range of $P_{t}$ ). Considering the linear behavior suggested by Eq. (3), we fit the data using $P_{t}=\alpha Q+\beta$ (red line), obtaining $\alpha=-k Q v / A=$ $-0.785 \mathrm{kPa} / \mathrm{g} \mathrm{s}^{-1}$ and $\beta=\sigma_{c}=1.87 \mathrm{kPa}$. Two important values are derived from this equation: when $Q=0$ (which is equivalent to building the material by adding grain by grain), it is found that the maximum cohesive stress for this liquid volume is $\sigma_{c}=1.87 \mathrm{kPa}$. On the other hand, when $P_{t}=0$, we have that $Q=Q_{\max }=2.4 \mathrm{~g} / \mathrm{s}$, which fixes a maximum value on $Q$ to build the sand towers. In fact, when this value is surpassed, a "bullet regime" is observed: the grains stick due to cohesion, but the vertical pressure produced by $Q$ is so high that the tower cannot grow. The grains form clusters at the base and they are ejected horizontally by the flux. This regime is shown for $Q=3.6 \mathrm{~g} / \mathrm{s}$ in movie 5 [17]. In Fig. 4(d) we calculated $\sigma_{c}=-\alpha Q+P_{t}$ as a function of $W$, for $Q=0.166 \mathrm{~g} / \mathrm{s}$. For $W=1, \sigma_{c}$ is minimum and increases as $W$ is reduced. A similar behavior of $\sigma_{c}$ in unsaturated materials have been recently reported [20,21]. Moreover, in the capillary regime, where the surface of the tower is basically a water-air interface, the Laplace pressure that holds the grains together can be approximated by $P=2 \gamma / \bar{r}_{g} \approx 400 \mathrm{~Pa}$. This value is similar to the cohesive stress measured in this regime, see $W \sim 0.9$ in Fig. 4(c).

According to [12], a cylindrical column of sand becomes unstable and buckles under its own weight when exceeding a critical height $h_{\text {crit }}=\left(9 J_{-1 / 3}^{2} G R_{T}^{2} / 16 \rho_{g} g\right)^{1 / 3}$ where $\rho_{g}$ is the density of the grains, $R_{T}$ the column radius, $g$ the gravitational acceleration, $J \approx 1.8663$ the smallest positive root of the Bessel function of order $-1 / 3$, and $G$ the elastic modulus. The optimum strength is given by $G=\alpha \bar{r}_{g}^{-1 / 3} E^{2 / 3} \gamma^{1 / 3}$, with $\bar{r}_{g}$ the radius of the grains, $E$ the Young's modulus of the grain material, and $\alpha=0.054$ a constant of proportionality related to the deformation of the capillary bonds. Thus, for a sand tower of $R_{T}=3 \mathrm{~mm}$ formed with sand grains of $\bar{r}_{g} \approx 10^{-4}$ $\mathrm{m}$ and $E=30 \mathrm{GPa}$, we found that the theoretical maximum height is $h_{\text {crit }}=150 \mathrm{~mm}$. Note in Fig. 2 that the highest towers found experimentally almost reached this maximum theoretical limit when the liquid volume was about $78 \%$. This is an intriguing result considering that the optimum strength is achieved at a very low liquid volume of $1 \%$ in Ref. [12]. Thus, this self-assembly process occurs in such a particular manner that ensures the perfect packing and arrangement of particles to obtain the maximum stability of the edifice.

Finally, we would like to mention a natural scenario that resembles the self-assembly mechanism and collapse reported 

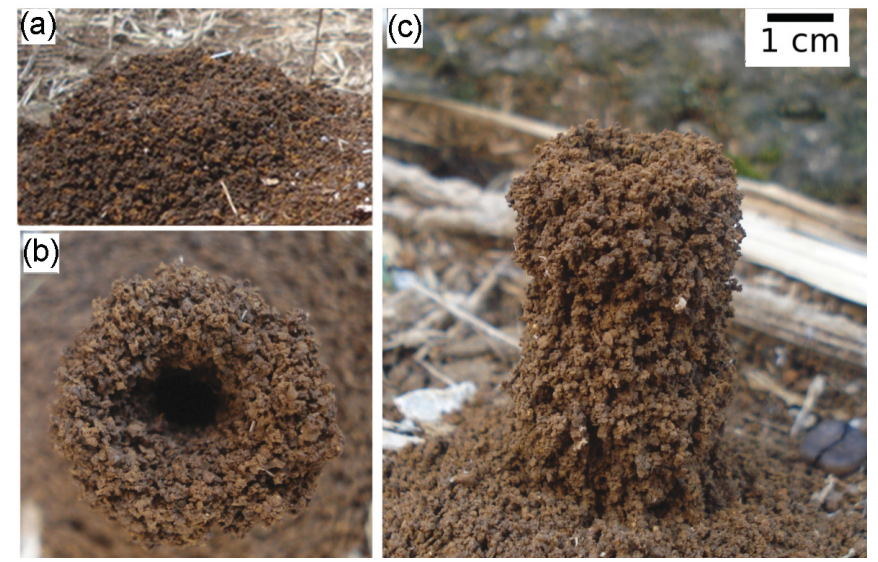

FIG. 5. (Color online) (a) Crater or mound formed by ants in a sunny day by passive deposition of grains around the nest. (b) Top and (c) lateral views of a granular tower formed by the same kind of ants by deposition of grains after a rain. Cohesion causes some grains to stick, forming the tower.

here. In Fig. 5 we show piles and towers formed by tropical ants in Mexico. In time of drought, the ants form a circular crater or pyramidal mound (usually of 100 to $150 \mathrm{~mm}$ in diameter) around the entrance hole. The formation results from a passive deposition, grain by grain, of excavated soil outside the nest. However, when the workers deposit the material after a rainfall, some grains stick due to cohesion. As a result, a spectacular tower grows on the wet surface. The only difference between both structures comes from the wetness conditions of the soil producing cohesion between the grains. Similarly, these towers reach around $100 \mathrm{~mm}$ in size.

\section{CONCLUSIONS}

Different morphologies are obtained when dry sand is poured onto a surface with different wetness conditions, as is schematically shown in Fig. 6. In particular, stable sand towers

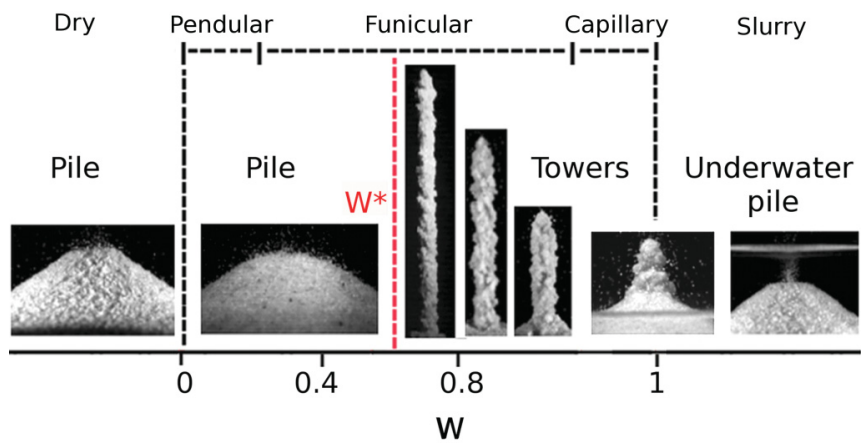

FIG. 6. (Color online) Different morphologies obtained by pouring dry grains on a flat surface depending on the liquid content of the substrate.

emerge when dry grains are poured on a wet granular bed in the funicular or capillary state, where the capillary rise of water is possible. The maximum height reached by the tower is linked to the limit of water imbibition into the structure and fixed by its volume fraction. On the other hand, an additional mechanical condition must be satisfied. The cohesive stress must balance the weight of the structure and the impulsion provided by the impacting grains. This mechanical limit is fixed by the local liquid content of the sand bed. These different ingredients explain the existence of a critical liquid content of the substrate below which the towers cannot arise. Moreover, that explains why the highest towers are found close to this limit because the cohesive stress is the largest for low liquid content values. Beyond an artistic technique to sculpt sandcastles, this is a new alternative to studying the mechanical properties of wet granular matter.

\section{ACKNOWLEDGMENTS}

The authors would like to thank FNRS and Conacyt Mexico for financial support. F.P.V. is a beneficiary of a grant from BELSPO/Marie Curie and the Université de Liège. F.P.V. thanks Conacyt Mexico, and S.D. thanks F.R.S.-FNRS for financial support. This work was also supported by the ODILE project (Contract No. FRFC 2.4623.11).
[1] H. M. Jaeger, C.-h. Liu, and S. R. Nagel, Phys. Rev. Lett. 62, 40 (1989).

[2] V. Frette, K. Christensen, A. Malthe-Sorenssen, J. Feder, T. Jossang, and P. Meakin, Nature 379, 49 (1996).

[3] E. Altshuler, O. Ramos, E. Martinez, A. J. Batista-Leyva, A. Rivera, and K. E. Bassler, Phys. Rev. Lett. 91, 014501 (2003).

[4] D. J. Hornbaker, R. Albert, I. Albert, A. L. Barabasi, and P. Schiffer, Nature 387, 765 (1997).

[5] T. C. Halsey and A. J. Levine, Phys. Rev. Lett. 80, 3141 (1998).

[6] A. Samadani and A. Kudrolli, Phys. Rev. E 64, 051301 (2001).

[7] S. Herminghauss, Adv. Phys. 54, 221 (2005).

[8] S. Nowak, A. Samadani, and A. Kudrolli, Nat. Phys. 1, 50 (2005); P. Schiffer, ibid. 1, 21 (2005).

[9] V. Richefeu, Moulay Saïd El Youssoufi, and F. Radjaï, Phys. Rev. E 73, 051304 (2006).
[10] M. Scheel, R. Seemann, M. Brinkmann, M. Di Michiel, A. Sheppard, B. Breidenbach, and S. Herminghaus, Nat. Mater. 7, 189 (2008); A. Kudrolli, ibid. 7, 174 (2008).

[11] N. Mitarai and F. Nori, Adv. Phys. 55, 1 (2006).

[12] M. Pakpour, M. Habibi, P. Moller, and D. Bonn, Sci. Rep. 2, 00549 (2012).

[13] D. M. Newitt and J. M. Conway-Jones, Trans. Inst. Chem. Eng. 36, 422 (1958).

[14] J. O. Marston, I. U. Vakarelski, and S. T. Thoroddsen, Phys. Rev. E 86, 020301(R) (2012).

[15] H. Li and J. J. McCarthy, Phys. Rev. Lett. 90, 184301 (2003).

[16] P. Tegzes, T. Vicsek, and P. Schiffer, Phys. Rev. E 67, 051303 (2003).

[17] See Supplemental Material at http://link.aps.org/supplemental/ 10.1103/PhysRevE.86.051303 for a detailed description of the experimental methods, data, and supplementary movies. The 
movies show the growth process of self-assembled sand towers over substrates with different regimes of liquid content. The description of the videos can be found in the README.txt file.

[18] J. Chopin and A. Kudrolli, Phys. Rev. Lett 107, 208304 (2011).
[19] J. Bragard and G. Lebon, Trans. Porous Med. 16(3), 253 (1994).

[20] N. Lu, T.-H. Kim, S. Sture, and W. J. Likos, J. Eng. Mech. 135, 1410 (2009).

[21] N. Vandewalle, G. Lumay, F. Ludewig, and J. E. Fiscina, Phys. Rev. E 85, 031309 (2012). 